\title{
Paradoxical Response after a Voriconazole Treatment in an Immunocompetent Host with a Skull Base Osteomyelitis due to Invasive Aspergillosis
}

\author{
Jeong-Min Kim, MD, PhD¹, Hae Bong Jung, MD¹, Jae-Han Bae, MD', Seong-Ho Choi, MD, PhD², Tae Jin Lee, MD, PhD³ \\ ${ }^{1}$ Department of Neurology, ${ }^{2}$ Division of Infectious Disease, Department of Internal Medicine, and ${ }^{3}$ Department of Pathology, Chung-Ang University Hospital, \\ Chung-Ang University College of Medicine, Seoul, Korea
}

Background: We describe a case of skull base osteomyelitis due to invasive aspergillosis which had been aggravated after antifungal treatment but significantly recovered by dexamethasone.

Case report: A 74-year-old male patient presented to neurology clinic complaining of sudden onset right-sided facial palsy and headache. Brain magnetic resonance imaging (MRI) and sphenoid sinus biopsy confirmed Aspergillus infection of skull base. He was treated with voriconazole for two months, but his headache was not relieved, and he additionally complained of vertigo and dysphagia. A subsequent MRI showed reduced enhancement of initial lesions, but increased thickness of surrounding dura mater. With an impression of paradoxical inflammatory response after antifungal treatment, parenteral dexamethasone was administered for one month while maintaining voriconazole. His symptoms improved thereafter

Conclusion: A paradoxical inflammatory response during antifungal treatment in the skull base aspergillosis aggravates the neurological symptom by thickening the dura mater, which can be recovered by dexamethasone.

J Neurocrit Care 2017;10(2):122-125

Key words: Neuroaspergillosis; Central nervous system infections
Received September 5, 2017

Revised September 28, 2017

Accepted September 30, 2017

Corresponding Author:

Tae Jin Lee, MD, PhD

Department of Pathology, Chung-

Ang University Hospital, Chung-

Ang University College of Medicine, 102 Heukseok-ro, Dongjak-gu, Seoul 06973, Korea

Tel: $+82-2-6299-2757$

Fax: +82-2-6293-5630

E-mail: taejlee@cau.ac.kr

Copyright $\odot 2017$ The Korean Neurocritical Care Society

\section{INTRODUCTION}

Invasive aspergillosis is a potentially lethal infection among an immunocompromised host; therefore, recent consensus guidelines emphasize the initiation of an antifungal agent and the reversal of immunosuppression, including recovery from neutropenia, or the reduction of an existing corticosteroid. ${ }^{1}$ However, an acute bacterial or tuberculous central nervous system infection can show a clinical worsening due to a paradoxical inflammatory response after an anti-microbial treatment and the combination of corticosteroid with an anti-microbial treatment could be helpful in these cases. ${ }^{2,3} \mathrm{~A}$ similar paradoxical response has also been reported in a fungal infection, and an immune reconstitution inflammatory syndrome can be diagnosed when a neutropenic patient experiences temporary worsening of symptoms not by uncontrolled infection but with recovery from neutropenia. ${ }^{4}$ We report a case of

cc This is an Open Access article distributed under the terms of the Creative Commons Attribution Non-Commercial License (http://creativecommons.org/licenses/by$\mathrm{nc} / 4.0$ ) which permits unrestricted non-commercial use, distribution, and reproduction in any medium, provided the original work is properly cited. 
an immunocompetent patient with a skull base osteomyelitis due to an invasive aspergillosis who experienced clinical aggravation with a characteristic magnetic resonance imaging (MRI) finding after an antifungal treatment, but recovered with from a combination of dexamethasone.

\section{CASE REPORT}

A 74-year-old man was admitted to the neurology clinic complaining of a sudden onset facial palsy, with hearing difficulty and a headache which had started seven days pre- viously. The throbbing headache involved the whole cranial area with a moderate to severe intensity and showed a partial response to tramadol. He complained of nausea and vomiting, but denied that he had dizziness or a febrile sense. He had been diagnosed with prostate cancer and had had surgical resection two years prior, but a relapse was detected four months previously and he underwent radiation therapy for two months. He did not have a prior history of diabetes mellitus or a human immunodeficiency virus infection. He did not undergo any immune modulating treatment.

On admission, he was alert and oriented, with a right
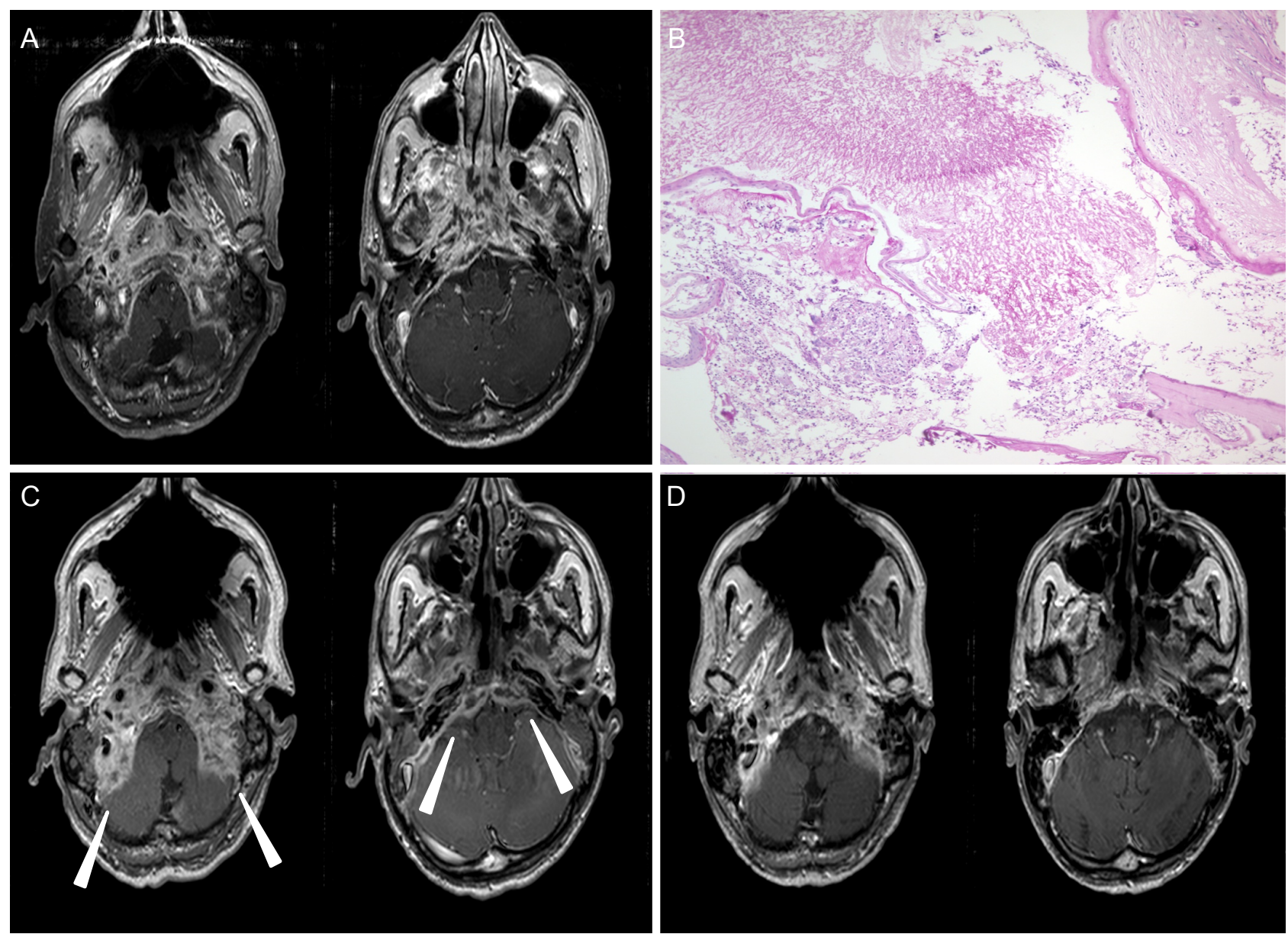

Figure 1. Brain magnetic resonance imaging (MRI) and pathological analysis findings. Initial brain MRI showed a diffuse enhancement of bony structures including the clivus and basis of occiput and sphenoid, as well as the posterior fossa dura, bilateral jugular foramen, carotid canals, pterygoid muscles and right sphenoid sinus (A). Hematoxylin and Eosin staining of mucosal specimen (magnification, $\times 40$ ) obtained from right sphenoid sinus revealed a large amount of non-pigmented septated hyphae with acute angle branching infiltrating mucosal layer, disclosing Aspergillus infection (B). A brain MRI performed two months after voriconazle treatment showed a decreased extent of the enhancement, but an increased thickness of the dura mater ( $C$, arrowheads). After a combination of dexamethasone, brain MRI followed after one month showed a markedly decreased dural thickness (D). 
side peripheral type facial palsy and bilateral sensorineuronal hearing loss. His vital signs were stable with a normal blood pressure of $130 / 80 \mathrm{mmHg}$ and a body temperature 36.5 degree Celsius. The initial blood test showed a mild neutrophil dominant leukocytosis of 11,640 per cubic millimeter (neutrophil percentage $=77 \%$ ) and an increased Creative protein $58.2 \mathrm{mg} / \mathrm{dL}$ (normal range $=0-10 \mathrm{mg} / \mathrm{dL}$ ) level. The renal and liver function tests were normal. An initial brain MRI showed a diffuse enhancing lesion involving the clivus and the base of the occiput and sphenoid bone, as well as a dura surrounding the posterior fossa, a bilateral jugular foramen, internal auditory canals, carotid canals and pterygoid muscles, with a focal mucosal enhancement in the right sphenoid sinus (Fig. 1A). A cerebrospinal fluid (CSF) study showed a normal opening pressure $\left(15 \mathrm{~cm} \mathrm{H}_{2} \mathrm{O}\right)$ with clear color, 9 white blood cells with elevated protein levels $(77.8 \mathrm{mg} / \mathrm{dL}$, normal range $=8-43$ $\mathrm{mg} / \mathrm{dL}$ ) and a normal glucose level $(65 \mathrm{mg} / \mathrm{dL}$, normal range $=40-70 \mathrm{mg} / \mathrm{dL}$, serum glucose: $113 \mathrm{mg} / \mathrm{dL}$ ), but negative results from a fungus staining and culture study. Pathologic analysis from the right sphenoid sinus confirmed the invasive aspergillosis (Fig. 1B). An intravenous voriconazole every 12 hours was followed by an oral agent of $200 \mathrm{mg}$ per day was initiated and maintained, and his hearing loss recovered gradually over two months, but his headache was not relieved. Moreover, he additionally complained of dysphagia and vertigo. A subsequent brain MRI showed a decreased enhancement over initial lesions, but an increased dural thickness (Fig. 1C). Repeated lumbar puncture tests showed one white blood cell and a markedly increased protein level $(209.2 \mathrm{mg} / \mathrm{dL})$ and a stable glucose level (62 mg/dL).

With an impression of a paradoxical response after an active antifungal treatment rather than infection progression, an administration of dexamethasone $5 \mathrm{mg}$ per every 6 hours was maintained for one month, and his symptoms markedly improved within 7 days. A subsequent brain MRI one month after the steroid combination revealed a markedly decreased dura thickness (Fig. 1D) and a CSF study showed a reduced protein level $(83.2 \mathrm{mg} / \mathrm{dL})$. He was discharged with $200 \mathrm{mg}$ of voriconazole and $2.5 \mathrm{mg}$ of pred- nisolone which was continued for 12 months. He denied the disease recurrence up to 37 months.

\section{DISCUSSION}

This patient suffered from an invasive Aspergillus infection involving a widespread skull base and the lower cranial nerves resulting in a headache, dysarthria, facial palsy, and hearing loss. Although an antifungal treatment reduced the disease activity, the transient neurological worsening, an increased dura thickening from a subsequent brain MRI and an elevated CSF protein suggested a paradoxical inflammatory reaction. The temporary combination of a corticosteroid dramatically reduced the symptom severity and dura thickness from the MRI. This is the first report describing the clinical and imaging characteristics of the paradoxical immune response after an antifungal treatment from an immunocompetent host with an invasive skull base aspergillosis.

Although Aspergillus infection has been commonly reported in an immunocompromised host which necessitates the prompt initiation of an antifungal treatment, there have been several case reports describing a chronic form of an invasive aspergillosis involving paranasal sinuses or orbits among immunocompetent hosts. ${ }^{5}$ The definition of an immune reconstitution inflammatory syndrome in patients with invasive aspergillosis can be made as clinical and radiological worsening with a robust decline of serum galactomannan values, and rising neutrophil counts. ${ }^{6} \mathrm{~A}$ paradoxical response after an effective antifungal treatment has been sufficiently described among patients with a pulmonary aspergillosis combined with a human immunodeficiency virus infection or neutropenia. ${ }^{7}$ A recent retrospective study among neutropenia patients with an invasive pulmonary aspergillosis showed a transient deterioration due to an immune reconstitution inflammatory syndrome occurred in $24 \%$ of the patients, which was more commonly related to the voriconazole treatment and to a favorable clinical course than those without this treatment. ${ }^{7}$ Serum Aspergillus galactomannan antigen titer is 
a useful test in differentiating an immune reconstitution inflammatory syndrome from an infection progression, which was not performed in this case. ${ }^{6}$ Future studies are warranted to identify the clinical and imaging characteristics of a paradoxical response among those patients with an invasive aspergillosis involving the central nervous system.

\section{Acknowledgements}

This study was supported by the Basic Science Research Program through the National Foundation of Korea (NRF) funded by the Ministry of Education, Science and Technology (NRF2016R1D1A1B03933891).

\section{REFERENCES}

1. Walsh TJ, Anaissie E), Denning DW, Herbrecht R, Kontoyiannis DP, Marr KA, et al. Treatment of aspergillosis: clinical practice guidelines of the Infectious Diseases Society of America. Clin Infect Dis 2008;46:327-60.

2. Thwaites GE, Nguyen DB, Nguyen HD, Hoang TQ, Do TT,
Nguyen TC, et al. Dexamethasone for the treatment of tuberculous meningitis in adolescents and adults. N Engl / Med 2004;351:1741-51.

3. Nguyen TH, Tran TH, Thwaites G, Ly VC, Dinh XS, Ho Dang $T N$, et al. Dexamethasone in Vietnamese adolescents and adults with bacterial meningitis. N Engl / Med 2007;357:2437-40.

4. Legris T, Massad M, Purgus R, Vacher-Coponat H, Ranque $\mathrm{S}$, Girard $\mathrm{N}$, et al. Immune reconstitution inflammatory syndrome mimicking relapsing cryptococcal meningitis in a renal transplant recipient. Transp/ Infect Dis 2011;13:303-8.

5. Pushker N, Meel R, Kashyap S, Bajaj MS, Sen S. Invasive aspergillosis of orbit in immunocompetent patients: treatment and outcome. Ophthalmology 2011;118:1886-91.

6. Miceli MH, Maertens J, Buvé K, Grazziutti M, Woods G, Rahman $M$, et al. Immune reconstitution inflammatory syndrome in cancer patients with pulmonary aspergillosis recovering from neutropenia: proof of principle, description, and clinical and research implications. Cancer 2007;110:112-20.

7. Jung J, Hong HL, Lee SO, Choi SH, Kim YS, Woo JH, et al. Immune reconstitution inflammatory syndrome in neutropenic patients with invasive pulmonary aspergillosis. I Infect 2015;70:659-67. 